\title{
Accelerated Particle Swarm Optimization to Solve Large-Scale Network Plan Optimization of Resource-Leveling with a Fixed Duration
}

\author{
Houxian Zhang ${ }^{1}{ }^{1}$ and Zhaolan Yang ${ }^{2}$ \\ ${ }^{1}$ School of Architecture and Civil Engineering, Nanjing Institute of Technology, Nanjing 211167, China \\ ${ }^{2}$ Industrial Center, Nanjing Institute of Technology, Nanjing 211167, China \\ Correspondence should be addressed to Houxian Zhang; houxianzhang@sina.com
}

Received 28 December 2017; Revised 18 March 2018; Accepted 20 March 2018; Published 16 May 2018

Academic Editor: Anna M. Gil-Lafuente

Copyright (C) 2018 Houxian Zhang and Zhaolan Yang. This is an open access article distributed under the Creative Commons Attribution License, which permits unrestricted use, distribution, and reproduction in any medium, provided the original work is properly cited.

\begin{abstract}
Large-scale network plan optimization of resource-leveling with a fixed duration is challenging in project management. Particle swarm optimization (PSO) has provided an effective way to solve this problem in recent years. Although the previous algorithms have provided a way to accelerate the optimization of large-scale network plan by optimizing the initial particle swarm, how to more effectively accelerate the optimization of large-scale network plan with PSO is still an issue worth exploring. The main aim of this study was to develop an accelerated particle swarm optimization (APSO) for the large-scale network plan optimization of resource-leveling with a fixed duration. By adjusting the acceleration factor, the large-scale network plan optimization of resourceleveling with a fixed duration yielded a better result in this study than previously reported. Computational results demonstrated that, for the same large-scale network plan, the proposed algorithm improved the leveling criterion by $24 \%$ compared with previous solutions. APSO proposed in this study was similar in form to, but different from, particle swarm optimization with contraction factor (PSOCF). PSOCF did not have as good adaptability as APSO for network plan optimization. Accelerated convergence particle swarm optimization (ACPSO) is similar in form to the APSO proposed in this study, but its irrationality was pointed out in this study by analyzing the iterative matrix convergence.
\end{abstract}

\section{Introduction}

The network plan is considered by the engineering community as a promising management method. A large-scale network plan with many works (such as more than 50) is an effective tool for solving large project management problems $[1,2]$. However, the number of possible solutions in largescale network plan optimization sharply increases with the number of works, and the time of calculation is exponential, far beyond the processing capacity of computing resources, so mathematics and computer science cannot solve the problem known as NP problem [2,3]. In recent years, genetic algorithm [4,5], Monte Carlo partition optimization [6], and particle swarm optimization (PSO) $[7,8]$ have provided an effective means to solve this problem.

PSO was proposed in 1995. Although the convergence of PSO is still controversial, its applied research has shown good results [9-13]. Experimental research includes optimization, biomedicine, communication, control, and so forth. Theoretical research includes PSO improvement, parameter selection, stability, convergence, and so forth. Improvement in performance of PSO reported in the literature included adjusting the parameters of PSO (inertial factor) [14-17], adopting the neighborhood topology [18], and combining with other algorithms (genetic algorithm, simulated annealing algorithm, and differential evolution algorithm) [19-22]. It does not include the solution to large-scale network plan optimization problems.

Accelerated optimization can be marked by betteroptimized solutions with the same number of iterations for iterative optimization. Yang et al. introduced some virtual particles in random directions with random amplitude to enhance the explorative capability of particles in PSO [23]; Qi et al. hybridized an improved estimation of distribution 
algorithm (EDA) using historic best positions to construct a sample space with PSO both in sequential and in parallel to improve population diversity control and avoid premature convergence for optimization of a water distribution network [24]; Zhang et al. added the random velocity operator from local optima to global optima into the velocity update formula of constriction particle swarm optimization (CPSO) to accelerate the convergence speed of the particles to the global optima and reduce the likelihood of being trapped into local optima [25]; Zhou et al. adjusted random functions with the density of the population so as to manipulate the weight of cognition part and social part and executed mutation on both personal best particle and group best particle to explore new areas [26]. Zhang and Yang accelerated the optimization of large-scale network plan resources and analyzed the acceleration optimization mechanism via stochastic process by optimizing the initial particle swarm using the Monte Carlo method under limiting conditions [7, 8, 27]; Ren and Wang proposed a PSO algorithm with accelerated convergence, theoretically proved the fast convergence of the algorithm, and optimized the parameters in the algorithm [28].

Inspired by previous efforts [28] to accelerate the convergence of PSO algorithm, this study proposed the method for the large-scale network plan optimization of resourceleveling with a fixed duration through debugging acceleration coefficient (it might also be described as accelerated PSO, or APSO for short) and yielded a better solution than reported in the previous literature.

This paper is organized as follows. Section 2 describes the experimental research of the large-scale network plan optimization of resource-leveling with a fixed duration using APSO. Section 3 analyzes the difference between APSO and PSO with a contraction factor (PSOCF) [29]. Section 4 analyzes the irrationality of accelerated convergence PSO (ACPSO) reported in [28].

\section{APSO to Solve the Large-Scale Network Plan Optimization of Resource-Leveling with a Fixed Duration}

Large-scale network plan optimization of resource-leveling with a fixed duration achieved the balance of resource demand in each period during the entire period of the project. Equilibrium could be marked by the variance of resources. The formula used to calculate the variance was as follows: $\sigma^{2}=\left(\sum_{i=1}^{J}\left(x_{i}-\mu\right)^{2}\right) / J$ where the total number of samples $x_{i}$ is $J$, and the arithmetic mean of $x_{i}$ is $\mu$. The smaller the variance, the more balanced the resource.

The evolutionary equation of basic PSO was as follows:

$$
\begin{aligned}
v_{i j}(t+1)= & w v_{i j}(t)+c_{1} \operatorname{rand}_{1}(t)\left(p_{g j}(t)-x_{i j}(t)\right) \\
& +c_{2} \operatorname{rand}_{2}(t)\left(p_{g}(t)-x_{i j}(t)\right) \\
x_{i j}(t+1)= & x_{i j}(t)+v_{i j}(t+1),
\end{aligned}
$$

where $t$ is the number of iterations; $x_{i j}(t+1)$ is $j$-dimension space coordinates of particle $i$ in $t+1$ iteration; $x_{i j}(t)$ is $j$ dimension space coordinates of particle $i$ in $t$ iteration; $w$ is inertial factor, usually taking the value of 1 according to the experience; $v_{i j}(t)$ is the $j$-dimension flight speed of particle $i$; $c_{1}$ and $c_{2}$ are accelerators evaluated usually between 0 and 2 by experience; rand $_{1}$ and rand $_{2}$ are random functions of value in the range of $[0,1] ; p_{g j}(t)$ is the best place to be experienced by particle $j$; and $p_{g}(t)$ is the best place for all particles to experience. The convergence condition was adopted by setting maximum iteration times $G$.

The evolutionary equation of accelerated PSO (APSO) was as follows:

$$
\begin{aligned}
& v_{i j}(t+1)=a\left(w v_{i j}(t)+c_{1} \operatorname{rand}_{1}(t)\left(p_{g j}(t)-x_{i j}(t)\right)\right. \\
& \left.\quad+c_{2} \operatorname{rand}_{2}(t)\left(p_{g}(t)-x_{i j}(t)\right)\right) \\
& x_{i j}(t+1)=x_{i j}(t)+v_{i j}(t+1),
\end{aligned}
$$

where $a$ is the acceleration coefficient, and the other signs are the same as earlier. The evolution equation of accelerated particle swarm algorithm has one more $a$ than with that of the basic PSO algorithm and one more $w$ than that of the particle swarm algorithm with contraction factor. However, it has produced significant results for solving large-scale network plan optimization of resource-leveling with a fixed duration as follows.

For example, a large network plan with 223 works is the same as Figure 1 in [27]. The debugging results of change $a$ are shown in Table 1, where the variance of the corresponding optimization results is 17.58 (better than the variance 22.99 quoted in [27]). The start time of each work is shown in Table 2, and the resource requirements of each unit time are shown in Table 3.

As shown in Table 1, for $w=1, c_{1}=c_{2}=2.05$, the number of particles 50 , and $G=100$, the minimum variance 17.58 could be obtained by adjusting the acceleration coefficient $a$, which was significantly optimized compared with the variance quoted in [27] without the acceleration coefficient (that is 22.99). For $w=1, c_{1}=3.5, c_{2}=0.4$, the number of particles 50 , and $G=100$, the minimum variance 18.4 could be obtained by adjusting the acceleration coefficient $a$, which was significantly optimized compared with the variance quoted in the literature [27]. For $w=0.8$, $c_{1}=c_{2}=2.05$, the number of particles 50 , and $G=100$, the minimum variance 18.93 could be obtained by adjusting the acceleration coefficient $a$, which was significantly optimized compared with the variance quoted in [27]. For $w=0.729$, $c_{1}=c_{2}=1.454$, the number of particles 50 , and $G=100$, variance smaller than 17.83 (acceleration coefficient 1) could not be obtained by adjusting the acceleration coefficient $a$.

\section{Difference between APSO and PSOCF [29]}

APSO proposed in this study was similar in form to PSOCF. The evolution equation of PSOCF was as follows [29]:

$$
\begin{aligned}
& v_{i j}(t+1)=\eta\left(v_{i j}(t)+c_{1} \operatorname{rand}_{1}(t)\left(p_{g j}(t)-x_{i j}(t)\right)\right. \\
& \left.\quad+c_{2} \operatorname{rand}_{2}(t)\left(p_{g}(t)-x_{i j}(t)\right)\right) \\
& x_{i j}(t+1)=x_{i j}(t)+v_{i j}(t+1),
\end{aligned}
$$


TABLE 1: Optimization parameter's debugging results of the large-scale network plan optimization of resource-leveling with a fixed duration using the accelerated particle swarm algorithm (particle number is $50 ; G=100$ ).

\begin{tabular}{|c|c|c|c|c|c|}
\hline sequence number & $w$ & $c_{1}$ & $c_{2}$ & $a$ & $\sigma^{2}$ \\
\hline 1 & 1 & 2.05 & 2.05 & 1 & 23.97 \\
\hline 2 & 1 & 2.05 & 2.05 & 0.33 & 19.31 \\
\hline 3 & 1 & 2.05 & 2.05 & 0.31 & 18.97 \\
\hline 4 & 1 & 2.05 & 2.05 & 0.3 & 17.58 \\
\hline 5 & 1 & 2.05 & 2.05 & 0.29 & 18.61 \\
\hline 6 & 1 & 2.05 & 2.05 & 3 & 31.43 \\
\hline 7 & 1 & 2.05 & 2.05 & 0.1 & 31.39 \\
\hline 8 & 1 & 2.05 & 2.05 & 0.03 & 31.43 \\
\hline 9 & 1 & 3.5 & 0.4 & 1 & 22.99 \\
\hline 10 & 1 & 3.5 & 0.4 & 0.03 & 31.43 \\
\hline 11 & 1 & 3.5 & 0.4 & 0.5 & 21.4 \\
\hline 12 & 1 & 3.5 & 0.4 & 0.8 & 31.43 \\
\hline 13 & 1 & 3.5 & 0.4 & 0.3 & 23.02 \\
\hline 14 & 1 & 3.5 & 0.4 & 0.6 & 16.35 error \\
\hline 15 & 1 & 3.5 & 0.4 & 0.4 & 20.8 \\
\hline 16 & 1 & 3.5 & 0.4 & 0.35 & 19.9 \\
\hline 17 & 1 & 3.5 & 0.4 & 0.33 & 18.4 \\
\hline 18 & 1 & 3.5 & 0.4 & 0.31 & 25.5 \\
\hline 19 & 0.8 & 2.05 & 2.05 & 1 & 24.53 \\
\hline 20 & 0.8 & 2.05 & 2.05 & 0.3 & 31.43 \\
\hline 21 & 0.8 & 2.05 & 2.05 & 1.2 & 31.43 \\
\hline 22 & 0.8 & 2.05 & 2.05 & 0.4 & 31.43 \\
\hline 23 & 0.8 & 2.05 & 2.05 & 0.5 & 31.00 \\
\hline 24 & 0.8 & 2.05 & 2.05 & 0.6 & 25.85 \\
\hline 25 & 0.8 & 2.05 & 2.05 & 0.7 & 18.93 \\
\hline 26 & 0.8 & 2.05 & 2.05 & 0.8 & 22.38 \\
\hline 27 & 0.729 & 1.454 & 1.454 & 1 & 17.83 \\
\hline 28 & 0.729 & 1.454 & 1.454 & 0.8 & 25.00 \\
\hline 29 & 0.729 & 1.454 & 1.454 & 1.05 & 20.54 \\
\hline 30 & 0.729 & 1.454 & 1.454 & 0.9 & 18.33 error \\
\hline
\end{tabular}

Note. The value in italics is the optimal value under certain parameter conditions.

where contraction factor $\eta=2 \kappa /|2-\varphi-\sqrt{\varphi(\varphi-4)}|, \kappa \in[0,1]$, $\varphi=c_{1}+c_{2}$. The other signs are the same as earlier.

For $c_{1}=3.5, c_{2}=0.4, \eta=2 \kappa /|2-\varphi-\sqrt{\varphi(\varphi-4)}|=2 \kappa / \mid 2-$ $3.9-\sqrt{3.9(3.9-4)} \mid$ does not exist. The PSOCF could not be used, but APSO in this study was used for optimization of network plan and the results were good, as shown in Table 1.

For $c_{1}=2.05, c_{2}=2.05, \eta=2 \kappa /|2-\varphi-\sqrt{\varphi(\varphi-4)}|=2(0 \sim$ 1) $/|2-4.1-\sqrt{4.1(4.1-4)}|=0 \sim 0.73$. The acceleration factor $a$ is outside the scope of the contraction factor $\eta$, and the optimization of APSO in this study was performed as usual, as shown in Table 1.

Thus, although, in this study, APSO was similar in form to PSOCF, essentially, for network plan optimization, PSOCF did not have as good adaptability as APSO.

\section{Irrationality of ACPSO Reported in [28]}

APSO proposed in this study was inspired by the ACPSO algorithm quoted in [28]. APSO was similar in form to
ACPSO. The evolution equation of ACPSO algorithm proposed in [28] was as follows:

$$
\begin{aligned}
& v_{i j}(t+1)=(\sin (\alpha))^{\beta}\left(w v_{i j}(t)\right. \\
& \quad+c_{1} \operatorname{rand}_{1}(t)\left(p_{g j}(t)-x_{i j}(t)\right) \\
& \left.+c_{2} \operatorname{rand}_{2}(t)\left(p_{g}(t)-x_{i j}(t)\right)\right) \\
& x_{i j}(t+1)=(\sin (\alpha))^{\beta} x_{i j}(t)+v_{i j}(t+1),
\end{aligned}
$$

where $\alpha$ is angle value with a distinct optimization effect when its value is within $[0, \pi / 8] ; \beta$ is a constant greater than zero, and the optimization effect is good when the value is 3 . The other signs are the same as earlier.

The ACPSO algorithm quoted in [28] was based on one inference: PSO is iterative. The iterative converges when the spectral radius of iterative matrix $L$ (that is the maximum absolute value of the matrix eigenvalue) is less than 1 . The smaller the spectral radius of $L$, the faster the iteration 
TABLE 2: The parameters and their optimization solution for the optimization example of the resource-leveling with a fixed duration.

\begin{tabular}{|c|c|c|c|c|c|}
\hline Number & Work & Duration & Resource Quantity & ES & Optimized ES \\
\hline 1 & $1-3$ & 2 & 2 & 0 & 0 \\
\hline 2 & $1-4$ & 2 & 1 & 0 & 0 \\
\hline 3 & $3-4$ & 2 & 1 & 2 & 2 \\
\hline 4 & $1-2$ & 1 & 2 & 0 & 0 \\
\hline 5 & $4-6$ & 4 & 1 & 4 & 3 \\
\hline 6 & $5-8$ & 4 & 1 & 7 & 7 \\
\hline 7 & $5-9$ & 5 & 1 & 7 & 8 \\
\hline 8 & $6-8$ & 5 & 3 & 13 & 13 \\
\hline 9 & $7-8$ & 8 & 3 & 15 & 15 \\
\hline 10 & $3-6$ & 3 & 2 & 2 & 3 \\
\hline 11 & $2-4$ & 3 & 2 & 1 & 1 \\
\hline 12 & $3-5$ & 5 & 1 & 2 & 2 \\
\hline 13 & $2-8$ & 3 & 2 & 1 & 1 \\
\hline 14 & $5-6$ & 6 & 2 & 7 & 6 \\
\hline 15 & $2-6$ & 1 & 3 & 1 & 1 \\
\hline 16 & $6-7$ & 2 & 0 & 13 & 12 \\
\hline 17 & $8-9$ & 7 & 3 & 23 & 22 \\
\hline 18 & $8-11$ & 2 & 2 & 23 & 25 \\
\hline 19 & $8-10$ & 2 & 1 & 23 & 23 \\
\hline 20 & $7-10$ & 2 & 1 & 15 & 15 \\
\hline 21 & $9-10$ & 1 & 2 & 30 & 29 \\
\hline 22 & $9-12$ & 4 & 1 & 30 & 28 \\
\hline 23 & $18-19$ & 8 & 3 & 57 & 57 \\
\hline 24 & $17-20$ & 2 & 1 & 51 & 52 \\
\hline 25 & $16-19$ & 4 & 1 & 49 & 48 \\
\hline 26 & $18-21$ & 5 & 1 & 57 & 58 \\
\hline 27 & $17-19$ & 5 & 3 & 51 & 51 \\
\hline 28 & $16-17$ & 2 & 2 & 49 & 49 \\
\hline 29 & $15-17$ & 2 & 1 & 46 & 46 \\
\hline 30 & $14-18$ & 2 & 1 & 42 & 41 \\
\hline 31 & $14-17$ & 1 & 2 & 42 & 41 \\
\hline 32 & $13-15$ & 2 & 1 & 44 & 44 \\
\hline 33 & $10-11$ & 4 & 1 & 31 & 30 \\
\hline 34 & $12-13$ & 5 & 1 & 39 & 39 \\
\hline 35 & $10-13$ & 5 & 3 & 31 & 30 \\
\hline 36 & $10-12$ & 8 & 3 & 31 & 30 \\
\hline 37 & $17-18$ & 6 & 0 & 51 & 51 \\
\hline 38 & $15-16$ & 3 & 2 & 46 & 46 \\
\hline 39 & $11-15$ & 5 & 1 & 35 & 34 \\
\hline 40 & $14-15$ & 3 & 2 & 42 & 41 \\
\hline 41 & $11-17$ & 5 & 1 & 35 & 35 \\
\hline 42 & $12-14$ & 3 & 2 & 39 & 38 \\
\hline 43 & $11-13$ & 6 & 2 & 35 & 34 \\
\hline 44 & $12-15$ & 2 & 0 & 39 & 38 \\
\hline 45 & $21-22$ & 2 & 2 & 70 & 69 \\
\hline 46 & $22-24$ & 2 & 1 & 75 & 88 \\
\hline 47 & $20-22$ & 2 & 1 & 73 & 85 \\
\hline 48 & $20-24$ & 1 & 2 & 73 & 85 \\
\hline 49 & $20-26$ & 2 & 1 & 73 & 84 \\
\hline
\end{tabular}


Mathematical Problems in Engineering

5

TABle 2: Continued.

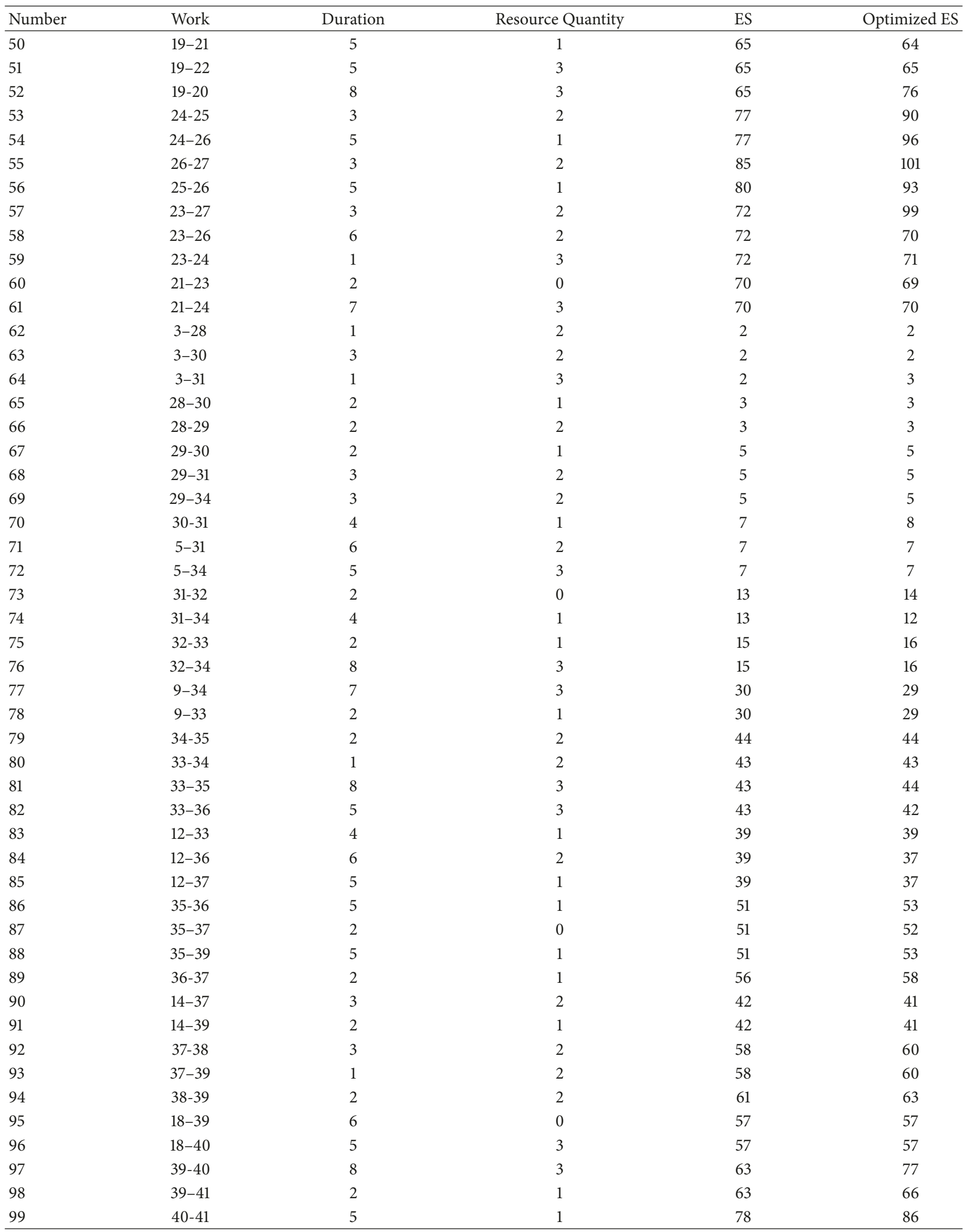


TABLe 2: Continued.

\begin{tabular}{|c|c|c|c|c|c|}
\hline Number & Work & Duration & Resource Quantity & ES & Optimized ES \\
\hline 100 & $40-42$ & 5 & 3 & 78 & 84 \\
\hline 101 & $21-40$ & 8 & 3 & 70 & 70 \\
\hline 102 & $21-42$ & 2 & 1 & 70 & 69 \\
\hline 103 & $21-43$ & 1 & 2 & 70 & 69 \\
\hline 104 & $41-42$ & 2 & 2 & 83 & 92 \\
\hline 105 & $41-43$ & 7 & 3 & 83 & 91 \\
\hline 106 & $41-45$ & 2 & 1 & 83 & 91 \\
\hline 107 & $42-43$ & 2 & 1 & 85 & 95 \\
\hline 108 & $23-43$ & 1 & 3 & 72 & 72 \\
\hline 109 & $23-45$ & 5 & 1 & 72 & 72 \\
\hline 110 & $43-44$ & 3 & 2 & 90 & 98 \\
\hline 111 & $43-45$ & 6 & 2 & 90 & 98 \\
\hline 112 & 44-45 & 5 & 1 & 93 & 101 \\
\hline 113 & $27-45$ & 3 & 2 & 88 & 104 \\
\hline 114 & $38-40$ & 4 & 1 & 61 & 63 \\
\hline 115 & $47-48$ & 2 & 2 & 8 & 9 \\
\hline 116 & $48-50$ & 2 & 1 & 10 & 11 \\
\hline 117 & $47-50$ & 2 & 1 & 8 & 9 \\
\hline 118 & $48-49$ & 1 & 2 & 10 & 11 \\
\hline 119 & $50-53$ & 4 & 1 & 14 & 16 \\
\hline 120 & $52-57$ & 4 & 1 & 49 & 49 \\
\hline 121 & $52-58$ & 5 & 1 & 49 & 61 \\
\hline 122 & $53-57$ & 5 & 3 & 55 & 56 \\
\hline 123 & $56-57$ & 8 & 3 & 57 & 59 \\
\hline 124 & $47-53$ & 3 & 2 & 8 & 9 \\
\hline 125 & $49-50$ & 3 & 2 & 11 & 12 \\
\hline 126 & $47-52$ & 5 & 1 & 8 & 8 \\
\hline 127 & $49-57$ & 3 & 2 & 11 & 13 \\
\hline 128 & $52-53$ & 6 & 2 & 49 & 50 \\
\hline 129 & $49-53$ & 1 & 3 & 11 & 12 \\
\hline 130 & $53-56$ & 2 & 0 & 55 & 56 \\
\hline 131 & $57-58$ & 7 & 3 & 65 & 68 \\
\hline 132 & $57-61$ & 2 & 2 & 65 & 68 \\
\hline 133 & $57-59$ & 2 & 1 & 65 & 67 \\
\hline 134 & $56-59$ & 2 & 1 & 57 & 58 \\
\hline 135 & $58-59$ & 1 & 2 & 72 & 75 \\
\hline 136 & $58-60$ & 4 & 1 & 72 & 76 \\
\hline 137 & $71-72$ & 8 & 3 & 99 & 102 \\
\hline 138 & $69-75$ & 2 & 1 & 93 & 98 \\
\hline 139 & $68-72$ & 4 & 1 & 91 & 94 \\
\hline 140 & $71-73$ & 5 & 1 & 99 & 103 \\
\hline 141 & $69-72$ & 5 & 3 & 93 & 98 \\
\hline 142 & $68-69$ & 2 & 2 & 91 & 95 \\
\hline 143 & $67-69$ & 2 & 1 & 88 & 92 \\
\hline 144 & $65-71$ & 2 & 1 & 84 & 88 \\
\hline 145 & $65-69$ & 1 & 2 & 84 & 87 \\
\hline 146 & $64-67$ & 2 & 1 & 86 & 90 \\
\hline 147 & $59-61$ & 4 & 1 & 73 & 76 \\
\hline 148 & $60-64$ & 5 & 1 & 81 & 85 \\
\hline
\end{tabular}


TABLE 2: Continued.

\begin{tabular}{|c|c|c|c|c|c|}
\hline Number & Work & Duration & Resource Quantity & ES & Optimized ES \\
\hline 149 & $59-64$ & 5 & 3 & 73 & 77 \\
\hline 150 & $59-60$ & 8 & 3 & 73 & 77 \\
\hline 151 & $69-71$ & 6 & 0 & 93 & 97 \\
\hline 152 & $67-68$ & 3 & 2 & 88 & 92 \\
\hline 153 & $61-67$ & 5 & 1 & 77 & 80 \\
\hline 154 & $65-67$ & 3 & 2 & 84 & 87 \\
\hline 155 & $61-69$ & 5 & 1 & 77 & 80 \\
\hline 156 & $60-65$ & 3 & 2 & 81 & 85 \\
\hline 157 & $61-64$ & 6 & 2 & 77 & 81 \\
\hline 158 & $60-67$ & 2 & 0 & 81 & 86 \\
\hline 159 & $73-76$ & 2 & 2 & 112 & 115 \\
\hline 160 & $76-79$ & 2 & 1 & 117 & 121 \\
\hline 161 & $75-76$ & 2 & 1 & 115 & 119 \\
\hline 162 & $75-79$ & 1 & 2 & 115 & 118 \\
\hline 163 & $75-83$ & 2 & 1 & 115 & 118 \\
\hline 164 & $72-73$ & 5 & 1 & 107 & 110 \\
\hline 165 & $72-76$ & 5 & 3 & 107 & 110 \\
\hline 166 & $72-75$ & 8 & 3 & 107 & 110 \\
\hline 167 & $79-82$ & 3 & 2 & 119 & 123 \\
\hline 168 & $79-83$ & 5 & 1 & 119 & 123 \\
\hline 169 & $83-84$ & 3 & 2 & 127 & 132 \\
\hline 170 & $82-83$ & 5 & 1 & 122 & 126 \\
\hline 171 & $78-84$ & 3 & 2 & 114 & 118 \\
\hline 172 & $78-83$ & 6 & 2 & 114 & 119 \\
\hline 173 & $78-79$ & 1 & 3 & 114 & 119 \\
\hline 174 & $73-78$ & 2 & 0 & 112 & 116 \\
\hline 175 & $73-79$ & 7 & 3 & 112 & 116 \\
\hline 176 & $46-47$ & 1 & 2 & 7 & 8 \\
\hline 177 & $47-51$ & 3 & 2 & 8 & 9 \\
\hline 178 & $47-54$ & 1 & 3 & 8 & 8 \\
\hline 179 & $46-51$ & 2 & 1 & 7 & 8 \\
\hline 180 & $29-46$ & 2 & 2 & 5 & 6 \\
\hline 181 & $29-51$ & 2 & 1 & 5 & 5 \\
\hline 182 & $29-54$ & 3 & 2 & 5 & 5 \\
\hline 183 & $51-54$ & 4 & 1 & 11 & 12 \\
\hline 184 & $52-54$ & 6 & 2 & 49 & 51 \\
\hline 185 & $34-52$ & 5 & 3 & 44 & 45 \\
\hline 186 & $54-55$ & 2 & 0 & 55 & 57 \\
\hline 187 & $34-54$ & 4 & 1 & 44 & 44 \\
\hline 188 & $55-62$ & 2 & 1 & 57 & 59 \\
\hline 189 & $34-55$ & 8 & 3 & 44 & 43 \\
\hline 190 & $34-58$ & 7 & 3 & 44 & 44 \\
\hline 191 & $58-62$ & 2 & 1 & 72 & 75 \\
\hline 192 & $34-62$ & 1 & 2 & 44 & 45 \\
\hline 193 & $35-62$ & 8 & 3 & 51 & 52 \\
\hline 194 & $62-63$ & 5 & 3 & 85 & 90 \\
\hline 195 & $60-62$ & 4 & 1 & 81 & 86 \\
\hline 196 & $60-63$ & 6 & 2 & 81 & 85 \\
\hline 197 & $60-66$ & 2 & 1 & 81 & 86 \\
\hline
\end{tabular}


TABle 2: Continued.

\begin{tabular}{|c|c|c|c|c|c|}
\hline Number & Work & Duration & Resource Quantity & ES & Optimized ES \\
\hline 198 & $35-63$ & 5 & 1 & 51 & 53 \\
\hline 199 & $35-66$ & 2 & 0 & 51 & 52 \\
\hline 200 & $63-66$ & 2 & 1 & 90 & 95 \\
\hline 201 & $65-66$ & 3 & 2 & 84 & 88 \\
\hline 202 & $39-65$ & 2 & 1 & 63 & 64 \\
\hline 203 & $66-70$ & 3 & 2 & 92 & 97 \\
\hline 204 & $39-66$ & 1 & 2 & 63 & 65 \\
\hline 205 & $39-70$ & 2 & 2 & 63 & 66 \\
\hline 206 & $39-71$ & 6 & 0 & 63 & 66 \\
\hline 207 & $71-74$ & 5 & 3 & 99 & 103 \\
\hline 208 & $39-74$ & 8 & 3 & 63 & 64 \\
\hline 209 & $41-74$ & 5 & 1 & 83 & 92 \\
\hline 210 & $74-77$ & 5 & 3 & 120 & 120 \\
\hline 211 & $73-74$ & 8 & 3 & 112 & 112 \\
\hline 212 & $73-77$ & 2 & 1 & 112 & 115 \\
\hline 213 & $73-80$ & 1 & 2 & 112 & 115 \\
\hline 214 & $41-77$ & 2 & 2 & 83 & 92 \\
\hline 215 & $41-80$ & 7 & 3 & 83 & 91 \\
\hline 216 & $77-80$ & 2 & 1 & 125 & 125 \\
\hline 217 & $78-80$ & 1 & 3 & 114 & 118 \\
\hline 218 & $45-78$ & 5 & 1 & 98 & 107 \\
\hline 219 & $80-81$ & 3 & 2 & 127 & 127 \\
\hline 220 & $45-80$ & 6 & 2 & 98 & 108 \\
\hline 221 & $81-84$ & 5 & 1 & 130 & 130 \\
\hline 222 & $45-84$ & 3 & 2 & 98 & 107 \\
\hline 223 & $70-74$ & 4 & 1 & 95 & 100 \\
\hline
\end{tabular}

Note. "ES" is the early start time of each work. "Optimized ES" is the start time optimized.

TABLE 3: The resource requirements of each unit time of large-scale network plan optimization of resource-levelling with a fixed duration using accelerated particle swarm algorithm (duration is 135).

\begin{tabular}{|c|c|c|c|c|c|c|c|c|c|c|c|c|c|c|c|c|c|}
\hline unit time & 1 & 2 & 3 & 4 & 5 & 6 & 7 & 8 & 9 & 10 & 11 & 12 & 13 & 14 & 15 & 16 & 17 \\
\hline resource requirements & 5 & 10 & 10 & 17 & 9 & 12 & 17 & 16 & 17 & 19 & 18 & 14 & 12 & 9 & 9 & 11 & 12 \\
\hline unit time & 18 & 19 & 20 & 21 & 22 & 23 & 24 & 25 & 26 & 27 & 28 & 29 & 30 & 31 & 32 & 33 & 34 \\
\hline resource requirements & 11 & 7 & 7 & 6 & 6 & 9 & 7 & 4 & 5 & 5 & 3 & 4 & 7 & 12 & 11 & 10 & 10 \\
\hline unit time & 35 & 36 & 37 & 38 & 39 & 40 & 41 & 42 & 43 & 44 & 45 & 46 & 47 & 48 & 49 & 50 & 51 \\
\hline resource requirements & 12 & 10 & 7 & 10 & 9 & 10 & 7 & 13 & 13 & 13 & 16 & 21 & 19 & 16 & 15 & 16 & 15 \\
\hline unit time & 52 & 53 & 54 & 55 & 56 & 57 & 58 & 59 & 60 & 61 & 62 & 63 & 64 & 65 & 66 & 67 & 68 \\
\hline resource requirements & 12 & 12 & 14 & 13 & 13 & 11 & 15 & 15 & 19 & 18 & 13 & 10 & 10 & 15 & 15 & 14 & 11 \\
\hline unit time & 69 & 70 & 71 & 72 & 73 & 74 & 75 & 76 & 77 & 78 & 79 & 80 & 81 & 82 & 83 & 84 & 85 \\
\hline resource requirements & 13 & 16 & 17 & 17 & 15 & 12 & 12 & 12 & 13 & 17 & 14 & 14 & 14 & 16 & 13 & 13 & 14 \\
\hline unit time & 86 & 87 & 88 & 89 & 90 & 91 & 92 & 93 & 94 & 95 & 96 & 97 & 98 & 99 & 100 & 101 & 102 \\
\hline resource requirements & 14 & 14 & 15 & 15 & 12 & 12 & 13 & 20 & 18 & 14 & 13 & 14 & 11 & 11 & 13 & 11 & 11 \\
\hline unit time & 103 & 104 & 105 & 106 & 107 & 108 & 109 & 110 & 111 & 112 & 113 & 114 & 115 & 116 & 117 & 118 & 119 \\
\hline resource requirements & 12 & 13 & 10 & 10 & 9 & 10 & 8 & 8 & 10 & 10 & 12 & 12 & 10 & 11 & 12 & 9 & 14 \\
\hline unit time & 120 & 121 & 122 & 123 & 124 & 125 & 126 & 127 & 128 & 129 & 130 & 131 & 132 & 133 & 134 & 135 & \\
\hline resource requirements & 15 & 11 & 9 & 9 & 8 & 8 & 4 & 3 & 4 & 3 & 3 & 2 & 1 & 3 & 3 & 3 & \\
\hline
\end{tabular}


converges. The absolute value of the eigenvalues of $L$ is as follows: $\left|\lambda_{1,2}\right|=\mid 1+w-\left(c_{1}+c_{2}\right) / 2 \pm\left(\left(-1-w+\left(c_{1}+\right.\right.\right.$ $\left.\left.\left.c_{2}\right) / 2\right)^{2}-4 w\right)^{1 / 2} / 2 \mid$, where $\sqrt{\left(-1-w+\left(c_{1}+c_{2}\right) / 2\right)^{2}-4 w} \geq$ 0 . The reasoning was problematic, and the analysis was as follows.

The evolution equation of PSO can be written in the matrix form:

$$
\left[\begin{array}{l}
v(t+1) \\
x(t+1)
\end{array}\right]=A_{t}\left[\begin{array}{l}
v_{t} \\
x_{t}
\end{array}\right]+\left[\begin{array}{ll}
\varphi_{1} & \varphi_{2} \\
\varphi_{1} & \varphi_{2}
\end{array}\right]\left[\begin{array}{l}
p_{i, t} \\
p_{g, t}
\end{array}\right],
$$

where $A_{t}=\left[\begin{array}{cc}w & -\varphi \\ w & 1-\varphi\end{array}\right], \varphi=\varphi_{1}+\varphi_{2}=c_{1} r_{1}+c_{2} r_{2}, p_{i, t}$ is the best place ever found, and $p_{g, t}$ is the best location for the whole particle swarm to date. The other signs are the same as earlier.

Set,

$$
\begin{aligned}
y(t) & =\left[\begin{array}{l}
v(t) \\
x(t)
\end{array}\right], \\
\phi_{t} & =\left[\begin{array}{l}
\varphi_{1} p_{i, t}+\varphi_{2} p_{g, t} \\
\varphi_{1} p_{i, t}+\varphi_{2} p_{g, t}
\end{array}\right] .
\end{aligned}
$$

Then, (5) can be changed to

$$
y(t+1)=A_{t} y(t)+\phi_{t} .
$$

$E$ is the mathematical expectation.

$$
\begin{aligned}
& E(y(t+1))=\left[\begin{array}{l}
E(v(t+1)) \\
E(x(t+1))
\end{array}\right], \\
& E\left(A_{t}\right)=\left[\begin{array}{cc}
w & -\frac{c_{1}+c_{2}}{2} \\
w & 1-\frac{c_{1}+c_{2}}{2}
\end{array}\right], \\
& E\left(\phi_{t}\right)=\left[\begin{array}{c}
\frac{c_{1} E\left(p_{i, t}\right)+c_{2} E\left(p_{g, t}\right)}{2} \\
\frac{c_{1} E\left(p_{i, t}\right)+c_{2} E\left(p_{g, t}\right)}{2}
\end{array}\right] .
\end{aligned}
$$

Set $Q_{t+1}=E(y(t+1)), M=E\left(A_{t}\right), Z=E\left(\phi_{t}\right)$. The characteristic value of $M$ is

$$
\begin{aligned}
& \lambda_{1,2} \\
& =\frac{1+w-\left(c_{1}+c_{2}\right) / 2 \pm \sqrt{\left(-1-w+\left(c_{1}+c_{2}\right) / 2\right)^{2}-4 w}}{2} .
\end{aligned}
$$
to be

As long as $1-\left(c_{1}+c_{2}\right) / 2 \neq-w \pm 2 \sqrt{w}$, matrix $A$ is going

$$
A M A^{-1}=L=\left[\begin{array}{cc}
\lambda_{1} & 0 \\
0 & \lambda_{2}
\end{array}\right]
$$

Set $A Q_{t+1}=H_{t}$. It could be deduced that the PSO algorithm was an iteration:

$$
H_{t+1}=L H_{t}+A Z \text {, }
$$

where $L$ is an iterative matrix.
The following two equations are equivalent to the inference in [30]:

$$
\begin{aligned}
A x & =b \\
x & =B x+g,
\end{aligned}
$$

where $A$ is the coefficient matrix, $x$ is the unknown column vector, $b$ is a constant number column vector, and $g$ is a constant matrix determined by $A$ and $b$.

The following iterative matrix $B$ could be obtained by the aforementioned system:

$$
x_{k+1}=B x_{k}+g .
$$

Set $x^{*}$ as the solution of the system. Then,

$$
x^{*}=B x^{*}+g \text {. }
$$

The aforementioned two formulas (13) and (14) on subtraction yield

$$
\begin{aligned}
x_{k+1}-x^{*} & =B\left(x_{k}-x^{*}\right)=B\left(B\left(x_{k-1}-x^{*}\right)\right) \\
& =B^{2}\left(x_{k-1}-x^{*}\right)=\cdots=B^{k+1}\left(x_{0}-x^{*}\right) .
\end{aligned}
$$

Because $x_{0}-x^{*}$ has nothing to do with $k, \lim _{k \rightarrow \infty}\left(x_{k+1}-\right.$ $\left.x^{*}\right)=0$ is equivalent to $\lim _{k \rightarrow \infty} B^{k+1}=0$. The theorem quoted in [30] shows $\lim _{k \rightarrow \infty} B^{k+1}=0$ equivalent to $\rho(B)<1$, where $\rho(B)$ is the spectral radius of matrix $B$.

Thus, the iterative matrix did not necessarily converge. Because the particle swarm algorithm did not have a set of equations to solve $Q_{t+1}$, the aforementioned reasoning could not be executed using the iterative matrix $L$ convergence.

In Table 1 , for $w=1, c_{1}=c_{2}=2.05$ (or $c_{1}=3.5, c_{2}=0.4$ ), the number of particles 50, and $G=100$, the acceleration coefficient $a=(\sin (\alpha))^{\beta}=\sin (\pi / 10)^{3}=0.03$ reflects the fact that the optimization of ACPSO in [28] was poor. This was the experimental verification of the problems of ACPSO quoted in [28].

\section{Conclusions}

This study proposed the method for the large-scale network plan optimization of resource-leveling with a fixed duration through adjusting the coefficient of APSO based on the algorithm quoted in [27] to obtain a better solution than previously reported. In other words, for the same large-scale network plan, the proposed algorithm improved the leveling criterion by $24 \%$ compared with previous solutions. Thus, the resource variances of 17.58 and 223 of a large-scale network plan are the best results for the large-scale network plan optimization of resource-leveling with a fixed duration to date in the literature.

Section 3 discusses the difference between APSO proposed in this study and PSOCF quoted in [29]. The proposed APSO was similar in form to PSOCF, but, essentially, PSOCF did not have as good adaptability as APSO for the network plan optimization.

Section 4 describes the difference between APSO proposed in this study and ACPSO quoted in [28]. Through 
analyzing the iterative matrix convergence of equations, it was pointed out that the derivation of iterative matrix convergence of ACPSO algorithm proposed in [28] was problematic, although it experimentally proved APSO was similar in form to ACPSO.

The effect of the APSO proposed in this study was verified to be obvious experimentally. However, the internal working mechanism of APSO is still a core issue worth investigation.

\section{Data Availability}

Data generated by the authors or analyzed during the study are available from the following options: (1) Data generated or analyzed during the study are available from the corresponding author by request. (2) All data generated or analyzed during the study are included in the published paper.

\section{Conflicts of Interest}

The authors declare that there are no conflicts of interest regarding the publication of this paper.

\section{References}

[1] E. L. Demeulemeester and W. S. Herroelen, Project Scheduling, Kluwer Academic Publishers, Boston, 2002.

[2] S. F. Li, K. J. Zhu, and D. Y. Wang, "Complexity study of the application of network plan technique to large project," Journal of China University of Geosciences (Social Science Edition), no. 9, pp. 90-94, 2010 (Chinese).

[3] X. F. Liu, Application Research of Network plan Technique Optimization Methods to Building Construction Management, Tianjin university, Tianjin, 2013.

[4] J.-L. Kim and J. R. D. Ellis, "Permutation-based elitist genetic algorithm for optimization of large-sized resource-constrained project scheduling," Journal of Construction Engineering and Management, vol. 134, no. 11, pp. 904-913, 2008.

[5] J.-W. Huang, X.-X. Wang, and R. Chen, "Genetic algorithms for optimization of resource Allocation in Large Scale Construction Project Management," Journal of Computers, vol. 5, no. 12, pp. 1916-1924, 2010.

[6] H. X. Zhang, "Resource-Leveling Optimization with Fixed Duration for a Large Network Plan Based on the Monte Carlo Method," Construction Technology, vol. 18, pp. 81-85, 2015.

[7] H. X. Zhang and Z. L. Yang, "Resource Optimization for a Large Network Plan on Particle Swarm Optimization," Mathematics in Practice and Theory, vol. 12, pp. 125-132, 2015.

[8] H. X. Zhang and Z. L. Yang, "Cost Optimization for a Large Network Plan Based on Particle Swarm Optimization," Mathematics in Practice and Theory, vol. 11, pp. 142-148, 2015.

[9] M. Wang and Q. Tian, "Dynamic heat supply prediction using support vector regression optimized by particle swarm optimization algorithm," Mathematical Problems in Engineering, vol. 2016, Article ID 3968324, 10 pages, 2016.

[10] F. Pan, W. X. Li, and Q. Gao, Particle Swarm Optimization and Multi-objective Optimization, Beijing Institute of Technology Press, 2013.

[11] A. Meng, Z. Li, H. Yin, S. Chen, and Z. Guo, "Accelerating particle swarm optimization using crisscross search," Information Sciences, vol. 329, pp. 52-72, 2016.
[12] Y. Fu, Z. L. Xu, and J. L. Cao, "Application of heuristic particle swarm optimization method in power network planning," Power System Technology, vol. 15, pp. 31-35, 2008.

[13] J. Sun, X. Wu, V. Palade, W. Fang, and Y. Shi, "Random drift particle swarm optimization algorithm: convergence analysis and parameter selection," Machine Learning, vol. 101, no. 1-3, pp. 345-376, 2015.

[14] A. Nickabadi, M. M. Ebadzadeh, and R. Safabakhsh, "A novel particle swarm optimization algorithm with adaptive inertia weight," Applied Soft Computing, vol. 11, no. 4, pp. 3658-3670, 2011.

[15] T. O. Ting, Y. Shi, S. Cheng, and S. Lee, "Exponential inertia weight for particle swarm optimization," Lecture Notes in Computer Science (including subseries Lecture Notes in Artificial Intelligence and Lecture Notes in Bioinformatics): Preface, vol. 7331, no. 1, pp. 83-90, 2012.

[16] Y.-T. Juang, S.-L. Tung, and H.-C. Chiu, "Adaptive fuzzy particle swarm optimization for global optimization of multimodal functions," Information Sciences, vol. 181, no. 20, pp. 4539-4549, 2011.

[17] A. Ismail and A. P. Engelbrecht, "The self-adaptive comprehensive learning particle swarm optimizer," Lecture Notes in Computer Science (including subseries Lecture Notes in Artificial Intelligence and Lecture Notes in Bioinformatics): Preface, vol. 7461, pp. 156-167, 2012.

[18] B. Y. Qu, J. J. Liang, and P. N. Suganthan, "Niching particle swarm optimization with local search for multi-modal optimization," Information Sciences, vol. 197, pp. 131-143, 2012.

[19] Y. Chen, D. Zhang, M. Zhou, and H. Zou, "Multi-satellite observation scheduling algorithm based on hybrid genetic particle swarm optimization," in Advances in Information Technology and Industry Applications, vol. 136 of Lecture Notes in Electrical Engineering, pp. 441-448, Springer, Berlin, Germany, 2012.

[20] S. Gholizadeh and F. Fattahi, "Serial integration of particle swarm and ant colony algorithms for structural optimization," Asian Journal of Civil Engineering, vol. 13, no. 1, pp. 127-146, 2012.

[21] A. Kaveh and S. Talatahari, "Particle swarm optimizer, ant colony strategy and harmony search scheme hybridized for optimization of truss structures," Computers \& Structures, vol. 87, no. 5-6, pp. 267-283, 2009.

[22] M. Khajehzadeh, M. R. Taha, A. El-Shafie, and M. Eslami, "Modified particle swarm optimization for optimum design of spread footing and retaining wall," Journal of Zhejiang University SCIENCE A, vol. 12, no. 6, pp. 415-427, 2011.

[23] Y. Yang, X. Fan, Z. Zhuo, S. Wang, J. Nan, and W. Chu, "Improved particle swarm optimization based on particles' explorative capability enhancement," Journal of Systems Engineering and Electronics, vol. 27, no. 4, pp. 900-911, 2016.

[24] X. Qi, K. Li, and W. D. Potter, "Estimation of distribution algorithm enhanced particle swarm optimization for water distribution network optimization," Frontiers of Environmental Science \& Engineering, vol. 10, no. 2, pp. 341-351, 2016.

[25] Z. Zhang, L. Jia, and Y. Qin, "Modified constriction particle swarm optimization algorithm," Journal of Systems Engineering and Electronics, vol. 26, no. 5, Article ID 07347871, pp. 1107-1113, 2015.

[26] Ch. H. Yang, W. H. Gui, and T. X. Dong, "A particle swarm optimization algorithm with variable random functions and mutation," Acta Automatica Sinical, vol. 7, pp. 1339-1347, 2014.

[27] H. Zhang and Z. Yang, "Large-Scale Network Plan Optimization Using Improved Particle Swarm Optimization Algorithm," 
Mathematical Problems in Engineering, vol. 2017, Article ID 3271969, 2017.

[28] Z. H. Ren and J. Wang, "Accelerate convergence particle swarm optimization algorithm," Control and Decision, vol. 2, pp. 201206, 2011.

[29] M. Clerc and J. Kennedy, "The particle swarm-explosion, stability, and convergence in a multidimensional complex space," IEEE Transactions on Evolutionary Computation, vol. 6, no. 1, pp. 58-73, 2002.

[30] D. S. H. Ma, N. Dong et al., Numerical calculation method, China Machine Press, 2015. 


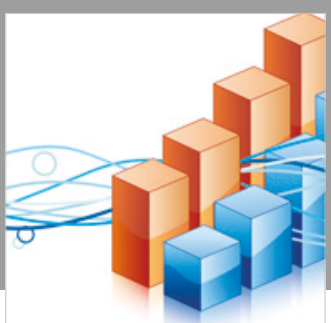

Advances in

Operations Research

\section{-n-m}
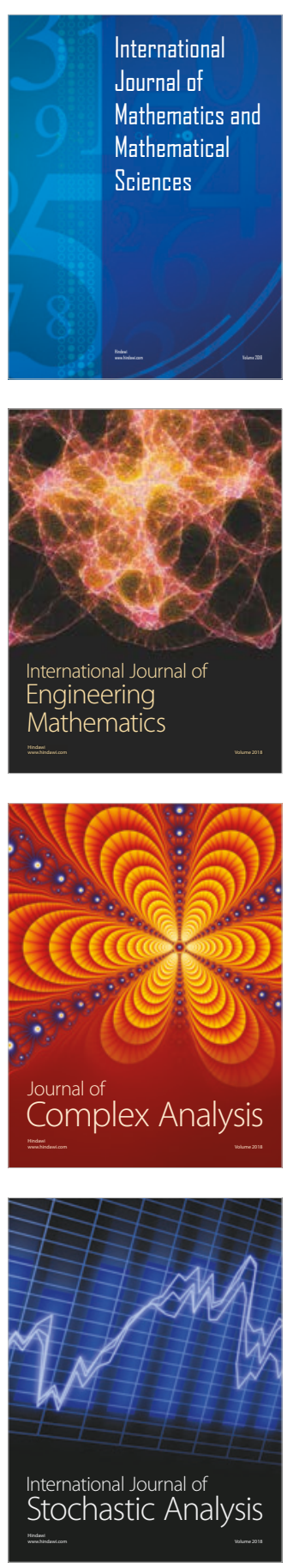
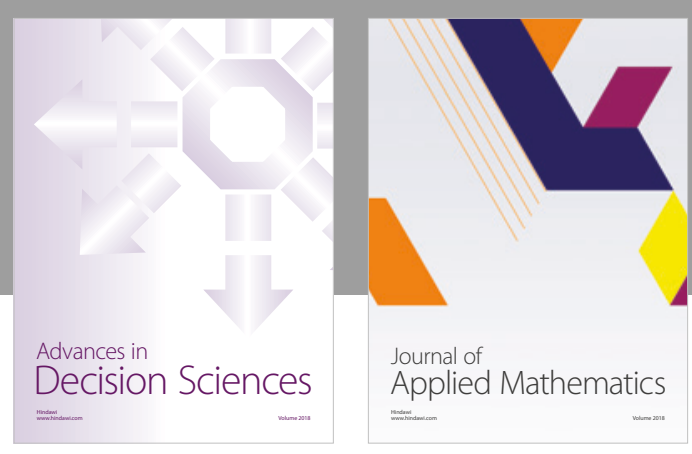

Journal of

Applied Mathematics
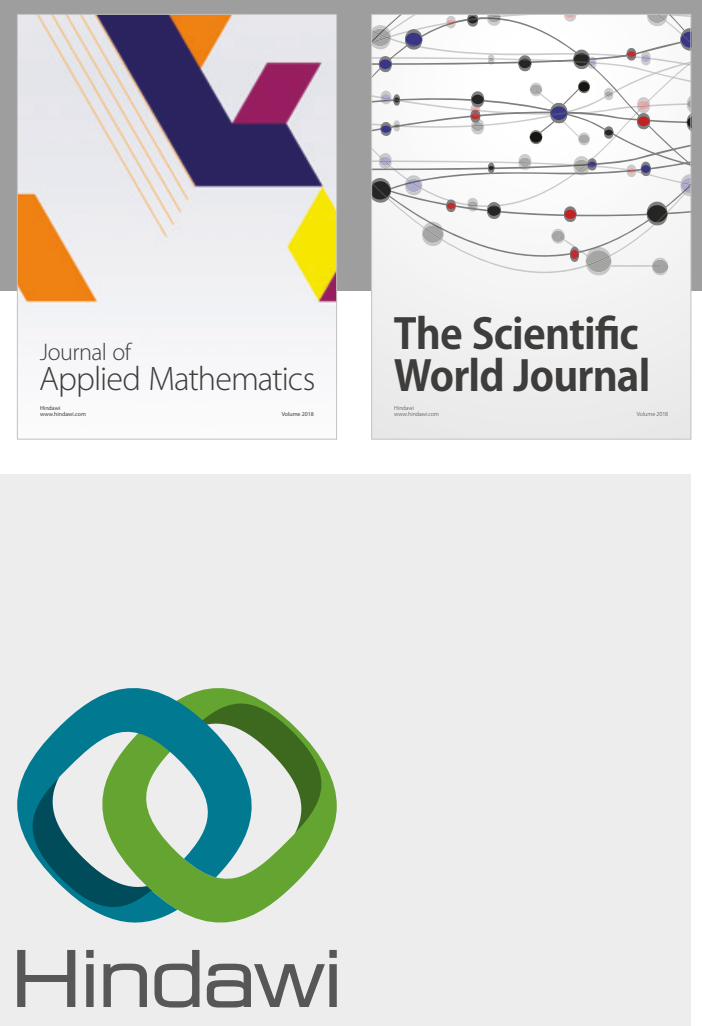

Submit your manuscripts at

www.hindawi.com

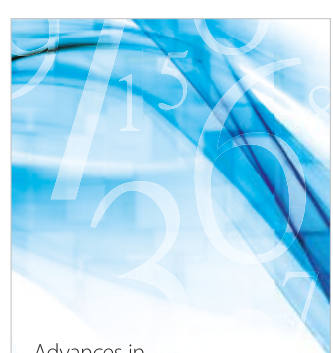

Advances in
Numerical Analysis
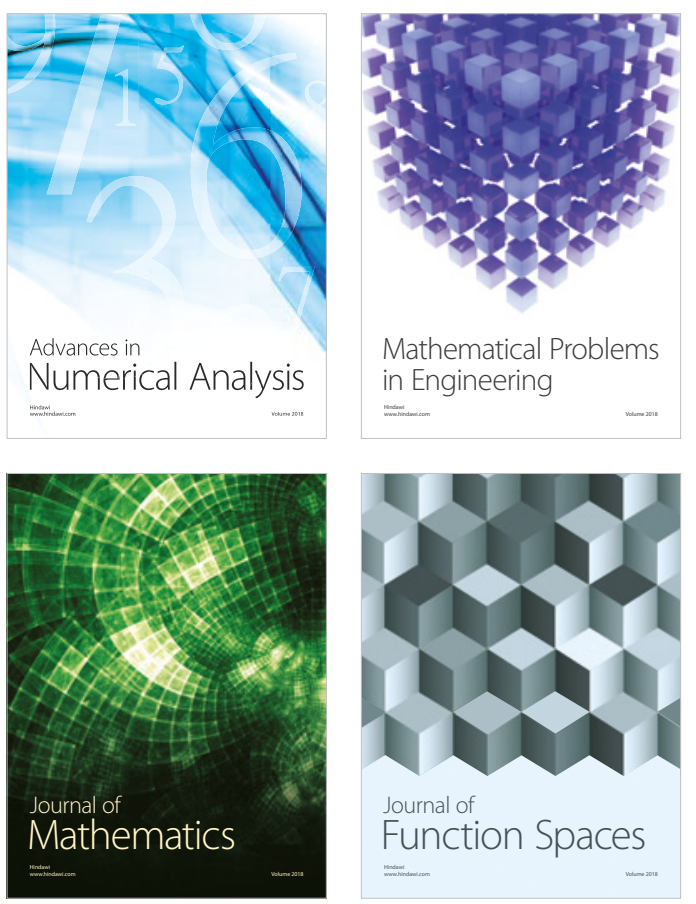

Mathematical Problems in Engineering

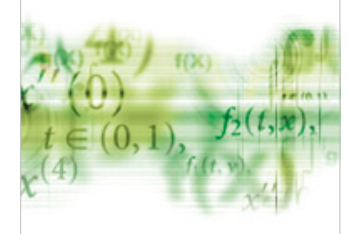

International Journal of

Differential Equations

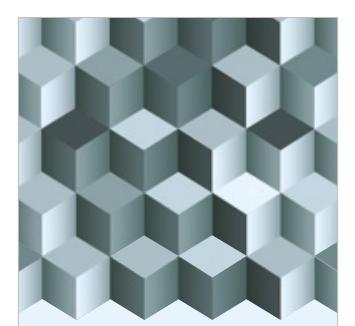

Journal of

Function Spaces

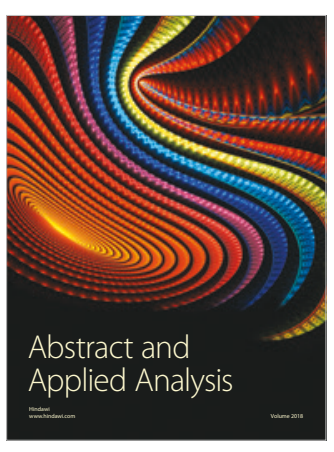

The Scientific

World Journal

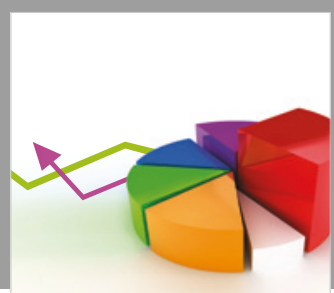

Journal of

Probability and Statistics
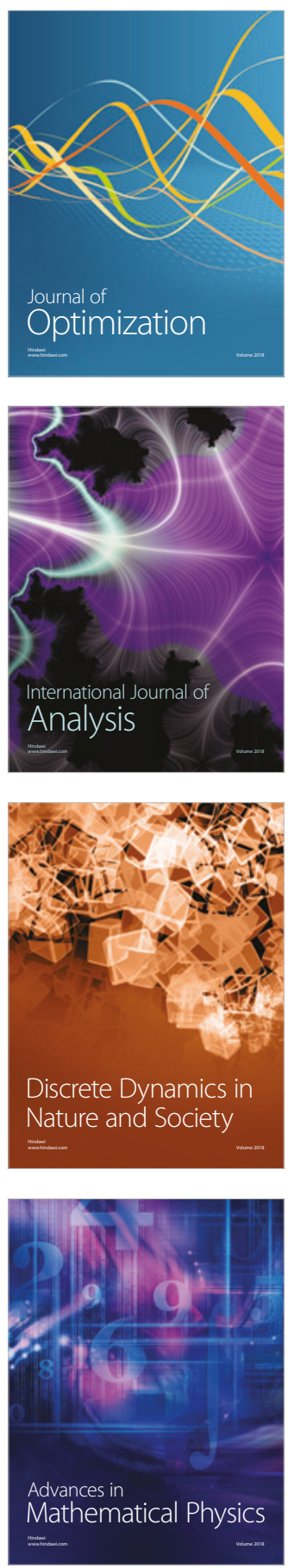